\title{
Repetitive Negative Thinking and Eating Disorders: A Meta-Analysis of the Role of Worry and Rumination
}

\author{
Sara Palmieri 1 1,2,3(D), Giovanni Mansueto ${ }^{2,3,4}$, Simona Scaini ${ }^{3}$, Gabriele Caselli 1,2,3, Walter Sapuppo ${ }^{1,2,3(\mathbb{D},}$ \\ Marcantonio M. Spada ${ }^{1} \oplus$, Sandra Sassaroli ${ }^{2,3}$ and Giovanni Maria Ruggiero ${ }^{2,3, *}$ \\ 1 Division of Psychology, London South Bank University, London SE1 0AA, UK; s.palmieri@milano-sfu.it (S.P.); \\ g.caselli@milano-sfu.it (G.C.); w.sapuppo@milano-sfu.it (W.S.); spadam@lsbu.ac.uk (M.M.S.) \\ 2 Studi Cognitivi, Cognitive Psychotherapy School and Research Center, 20132 Milan, Italy; \\ g.mansueto@milano-sfu.it (G.M.); s.sassaroli@milano-sfu.it (S.S.) \\ 3 Department of Psychology, Sigmund Freud University, 20143 Milan, Italy; s.scaini@milano-sfu.it \\ 4 Department of Health Sciences, University of Florence, 50121 Florence, Italy \\ * Correspondence: gm.ruggiero@milano-sfu.it; Tel.: +39-02-8324185
}

\section{check for} updates

Citation: Palmieri, S.; Mansueto, G.; Scaini, S.; Caselli, G.; Sapuppo, W.; Spada, M.M.; Sassaroli, S.; Ruggiero, G.M. Repetitive Negative Thinking and Eating Disorders: A

Meta-Analysis of the Role of Worry and Rumination. J. Clin. Med. 2021, 10, 2448. https://doi.org/ $10.3390 / \mathrm{jcm} 10112448$

Academic Editors: Yael Latzer and Elena Tomba

Received: 30 March 2021

Accepted: 24 May 2021

Published: 31 May 2021

Publisher's Note: MDPI stays neutral with regard to jurisdictional claims in published maps and institutional affiliations.

Copyright: (c) 2021 by the authors. Licensee MDPI, Basel, Switzerland. This article is an open access article distributed under the terms and conditions of the Creative Commons Attribution (CC BY) license (https:/ / creativecommons.org/licenses/by/ $4.0 /)$.

\begin{abstract}
The role of worry and rumination in eating disorders (EDs) is controversial. This metaanalysis of the literature is aimed at clarifying the relationship between repetitive negative thinking (RNT) and EDs. In accordance with the PRISMA criteria, a comprehensive search of the literature was conducted on PubMed and PsycInfo from inception to March 2021. Search terms: "eating disorder/anorexia/bulimia/binge eating disorder" AND “worry/rumination/brooding/repetitive thinking". A manual search of reference lists was also run. Forty-three studies were included. RNT was found to be associated with anorexia, bulimia, and binge eating disorder. A moderating effect was found for "presence/absence ED diagnosis" and "subtype of ED symptom". ED patients showed higher RNT than the general population. No differences were observed for age or between worry and rumination in the magnitude of their association with EDs.
\end{abstract}

Keywords: repetitive negative thinking; rumination; worry; eating disorder; anorexia; bulimia; binge eating disorder

\section{Introduction}

Repetitive negative thinking (RNT) is a cognitive process characterised by a repetitive, frequent, and self-focused form of thinking [1]. Worry and rumination have been grouped under the construct of RNT [2,3]. Worry has been defined as a chain of thoughts and images laden with negative affects and relatively uncontrollable [4]. Worry is an attempt to engage in mental problem-solving on an issue whose outcome is unknown but contains the possibility of being negative. Rumination is defined as thoughts that repetitively focus attention on negative emotions and symptoms, their causes, meanings, and consequences [5]. Rumination can take verbal and imaginary forms [6], characterized by the tendency to repeatedly think on the self, upsetting events, and personal concerns [7]. Worry is usually focused on problem-solving and is more future-oriented, whereas rumination often consists of themes of loss and typically has a focus on past problems [8].

An extensive literature base has suggested that both worry and rumination are cognitive processes present across diverse disorders [2]. Worry is associated with anxiety disorders [9-12] and major depressive disorder [13-15]. Rumination is associated with both the development and persistence of mood and anxiety disorders [16-24], addictive behaviours [25,26], and schizophrenia [27].

It is extensively acknowledged that preoccupation with the control of eating, weight, and shape is conceptualized as core feature of eating disorder (ED) psychopathology [28,29]. Thus, it is conceivable that individuals presenting with EDs may report a stronger tendency to engage in RNT. A recent meta-analysis by Smith, Mason and Lavender [30] pointed out 
that rumination is concurrently and prospectively associated with ED psychopathology and that individuals with EDs showed higher levels of rumination than those without an ED. Although the association between rumination and EDs has been explored, no study has systematically examined the role of worry in EDs. Notwithstanding some evidence showing raised levels of worry in patients with EDs compared to controls from the general population [31-33], it is not possible to draw a conclusion about the association between worry and EDs due to the lack of a systematic review or meta-analysis.

Thus, an increasing number of studies have explored the association between RNT and EDs, and the relationship between rumination and EDs has been highlighted in the metaanalysis by Smith and colleagues [30]. However, to date, no qualitative or quantitative reviews have been performed that take into account both worry and rumination and possible moderators of the relationship between RNT and EDs. Clarifying this relationship could have implications for clinical practice, especially with respect to interventions aimed at interrupting RNT.

Through the use of meta-analytic techniques, the present study aimed to present a comprehensive evaluation of the literature on EDs, worry and rumination in order to: (1) extend the literature and estimate the magnitude of the association between EDs, worry and rumination; (2) explore the role of some moderators such (a) "subtypes of ED symptoms", the (b) "presence vs. absence of a diagnosis of ED" and (c) "worry vs. rumination" and (3) "mean age of the sample" in shaping heterogeneity.

\section{Materials and Methods}

\subsection{Study Selection}

The methodology of the study selection will be reported in accordance with the Preferred Reporting Items for Systematic Reviews and Meta-Analyses (PRISMA) guidelines [34].

\subsubsection{Eligible Studies Included}

The inclusion criteria applied to the literature search were: (a) English language articles published in peer-reviewed journals; (b) diagnosis of ED determined according to the standardized diagnostic criteria, including the Diagnostic and Statistical Manual of Mental Disorders (DSM) from the third to the fifth edition [35-39], the Research Diagnostic Criteria (RDC) [40] or the International Classification of Diseases (ICD) from the sixth to the tenth edition [41-45] and assessing worry and/or rumination; (c) studies using a case-control design/prospective cohort studies/large population-based cross-sectional studies/experimental studies and (d) information available to determine the effect size.

Studies including participants with a diagnosis of neurological and/or neurocognitive organic impairment, or co-occurrent psychiatric disorders or obese participants, were not included, as well as studies on cognitive processes not specifically referring to worry and rumination.

\subsubsection{Information Sources and Search}

PubMed and PsycInfo were systematically searched from inception to 31 March 2021. Furthermore, a manual search of reference lists from all the articles selected, full textreviews and significant reviews was run. Search terms included: eating disorder/anorexia/ bulimia/binge eating disorder combined using Boolean "AND" operator with worry/ rumination/brooding/repetitive thinking.

\subsubsection{Study Selection, Data Collection Process and Data Items}

The studies' eligibility was assessed through the following procedure: title screening, abstract screening and full paper screening. Titles and abstracts were screened by S.P. Articles appearing potentially relevant were retrieved by S.P. and independently assessed by S.P. and G.M. Consistent with the previous studies [46-48], disagreements on eligibility were resolved by consensus among authors (intercoder reliability: Cohen's Kappa coeffi- 
cient $=0.70$ ). The following assumptions were made: if not specified, participants were considered without co-occurrent psychiatric disorders or neurological or neurocognitive organic impairment.

\subsubsection{Assessment of Risk of Bias in Individual Studies}

In order to ascertain the validity of the eligible studies, two investigators independently rated each study on the basis of the following markers: homogeneity of the sample regarding the diagnosis if present, appropriateness of random allocation if necessary and presence of a comparable group if appropriate. Disagreements were resolved by consensus [46-48] (intercoder reliability: Cohen's Kappa coefficient $=0.80$ ).

\subsection{Quality Assessment}

In accordance with previous studies [46-49], the quality of each eligible study was assessed independently by two investigators (S.P. and G.M.) using the Newcastle Ottawa scale for case-control studies and its adapted form for cross-sectional studies [49,50]. Disagreements were resolved by consensus (intercoder reliability: Cohen's Kappa coefficient $=0.85$ ).

\subsection{Data Analyses}

Analyses were conducted using Comprehensive Meta-analysis, CMA version 2.0 by Biostat. Using a random effects model, we calculated the effect size (ES), which was reported here as the standardized difference between the means of the two groups (Cohen's d), together with their 95\% CIs. According to Cohen's criteria (Cohen, 1988), an ES of $<0.20$ is considered a small effect, an ES of about 0.50 is a moderate effect and ES of about 0.80 is a large effect. For the purposes of the current study, a positive ES indicated an association between RNT and eating problems. Each ES was calculated for each self-report symptoms scale included in the identified studies and averaged across measures to obtain an ES for each study [51,52].

In order to address the publication bias, i.e., the possibility that published studies have larger mean ES than unpublished studies, we checked the results using the "Trim and Fill" procedure [53] and the Classic Fail-safe Number method [54]. The "Trim and Fill" procedure is a nonparametric method that evaluates the effect of potential data censoring on the meta-analysis [53]. Using this method, a plot of each study's ESs against the meta-sample's ES and standard error was built. These plots should be shaped as a funnel when no data censoring is present. Since smaller or nonsignificant studies are less likely to be published, studies in the bottom left/right-hand corner of the plot are often omitted [55]. The most symmetrically right/left unmatching studies in the meta-analysis are thus trimmed and replaced with their missing counterparts imputed or "filled" in as mirror images of the trimmed outcomes [56]. This allows for the computation of an adjusted ES and relative CI. The Classic Fail-safe Number estimates the number of studies with nonsignificant findings, which are necessary to make the combined ES nonsignificant [56]. Meta-analyses with a fail-safe number higher than $\left(5^{*}\right.$ studies number +10$)$ are usually considered free from publication bias [54].

The presence of heterogeneity across the studies was evaluated by the $\mathrm{I}^{2}$ index, which measures the proportion of total variation due to real differences in the variability of ESs among studies [56]. The $Q$ statistic was used to test the heterogeneity of the specific set of ESs and the effects of the selected moderators [56]. We considered the following moderators: (a) subtypes of ED symptoms (anorexia nervosa (AN), bulimia nervosa (BN) and binge eating disorder (BED)); (b) presence vs. absence of any diagnosis of EDs (i.e., the comparison between patients and healthy controls from the population); (c) subtype of RNT: worry or rumination and (d) mean age of the sample.

The 1st and 2nd authors independently coded the qualitative moderators in each study, and they reached consensus in the case of disagreements. No disagreements were found among the authors. 
For dichotomous moderators, we carried out a subgroup analysis based on a mixedeffect model, assuming a common among-study variance component across the subgroups and a random effect model to combine the subgroups. A Q-test was used to test for heterogeneity across the subgroups [56].

\section{Results}

\subsection{Study Selection}

The search of the PubMed and PsycInfo databases and the manual search showed a total of 1570 citations. After removing the duplicates and reviewing the abstracts to exclude those that clearly did not meet the criteria $(n=620), 89$ remained. Of these, 34 studies were excluded, because they did not meet the inclusion criteria. Of the 55 studies that remained, 12 studies were further discarded. Figure 1 illustrates the search and screening process. The 43 articles that met the inclusion criteria are listed in Tables 1 and 2, along with their study characteristics. The total sample size of the selected studies comprised 10,391 participants; among them, 1345 were $\mathrm{ED}$ cases $(\mathrm{AN}=521, \mathrm{BN}=278, \mathrm{BED}=325$ and $\mathrm{EDNOS}=221$ ), and 9046 were healthy controls from the general population. The following is a summary of the literature concerning the association between ED, worry and rumination.

A total of 43 studies, 10 on worry and 35 on rumination, were identified for inclusion in the meta-analysis. Thirteen reports were considered more than one time, since some studies included both AN and BN samples [32,33,57-61] or included clinical and general populations [62-64] or included different kinds of general populations [65] or included both worry and rumination $[57,66]$, leaving a total of 57 entries for the meta-analysis.

Table 1. Summary of the demographic characteristics of the studies assessed and the relationship between worry and eating behavior.

\begin{tabular}{|c|c|c|c|c|c|c|c|}
\hline Source & Study Design & Sample-Size & $\begin{array}{l}\text { Age (Years) } \\
\text { Mean } \pm \text { SD }\end{array}$ & Sex $\%(n)$ & $\begin{array}{l}\text { Diagnostic Tool } \\
\text { Eating Measure }\end{array}$ & $\begin{array}{l}\text { Sub-Types of } \\
\text { ED Symptoms }\end{array}$ & Worry Measure \\
\hline $\begin{array}{l}\text { Napolitano } \\
\text { and Himes } \\
2011[31]\end{array}$ & case-control & $\begin{array}{l}\text { cases: } 46 \\
\text { binge eating } \\
\text { group without } \\
\text { BED: } 186\end{array}$ & - & $\begin{array}{l}\mathrm{F}: 100 \% \\
(232)\end{array}$ & $\begin{array}{l}\text { DSM-IV-TR } \\
\text { EDDS }\end{array}$ & BED & FOBES \\
\hline $\begin{array}{l}\text { Sassaroli et al. } \\
\text { 2005 [32] }\end{array}$ & case-control & $\begin{array}{l}\text { cases: } 63 \\
\text { controls: } 30\end{array}$ & $\begin{array}{c}23.06 \pm 4.54 \text { vs. } \\
26.32 \pm 4.27\end{array}$ & $\begin{array}{c}\text { F: } 100 \% \\
(93)\end{array}$ & SCID-I & $\begin{array}{l}\mathrm{AN} \\
\mathrm{BN}\end{array}$ & PSWQ \\
\hline $\begin{array}{l}\text { Sternheim } \\
\text { et al. } 2012 \text { [33] }\end{array}$ & case-control & $\begin{array}{c}\text { cases: } 45 \\
\text { controls: } 37\end{array}$ & $\begin{array}{c}25.8 \pm 8.5 \text { vs. } \\
27.8 \pm 10.2\end{array}$ & not reported & $\begin{array}{l}\text { DSM-IV } \\
\text { EDE }\end{array}$ & $\begin{array}{l}\mathrm{AN} \\
\mathrm{BN}\end{array}$ & $\begin{array}{c}\text { PSWQ } \\
\text { Catastrophizing } \\
\text { Interview }\end{array}$ \\
\hline $\begin{array}{c}\text { González et al. } \\
2017 \text { [57] }\end{array}$ & cross-sectional & $\begin{array}{c}\text { general } \\
\text { population: } 176\end{array}$ & $31.2 \pm 13.3$ & F: 67\% (118) & EAT & $\begin{array}{l}\mathrm{AN} \\
\mathrm{BN}\end{array}$ & PSWQ \\
\hline $\begin{array}{l}\text { Kollei, et al. } \\
2012 \text { [58] }\end{array}$ & case-control & $\begin{array}{l}\text { cases: } 66 \\
\text { control: } 33\end{array}$ & $\begin{array}{l}\text { AN: } 26.94 \pm 9.15 \\
\text { BN: } 25.94 \pm 8.25 \\
\text { CG: } 26.91 \pm 8.48\end{array}$ & $\begin{array}{c}\text { F: } 95.45 \% \\
\quad(63) \\
\text { vs. } 69.7 \% \\
\quad(23)\end{array}$ & DSM-IV & $\begin{array}{l}\mathrm{AN} \\
\mathrm{BN}\end{array}$ & CITQ \\
\hline $\begin{array}{l}\text { Sapuppo et al. } \\
2018 \text { [60] }\end{array}$ & case-control & $\begin{array}{c}\text { cases: } 84 \\
\text { controls: } 38\end{array}$ & $\begin{array}{c}23.39 \pm 4.75 \text { vs. } \\
25.31 \pm 5.4\end{array}$ & $\begin{array}{l}\text { F: } 100 \% \\
(122)\end{array}$ & SCID-I & $\begin{array}{l}\mathrm{AN} \\
\mathrm{BN}\end{array}$ & PSWQ \\
\hline $\begin{array}{l}\text { Startup et al. } \\
2013 \text { [66] }\end{array}$ & cross-sectional & cases: 62 & $26.6 \pm 7.8$ & $\begin{array}{c}\text { F: } 93.5 \% \\
\quad(58)\end{array}$ & $\begin{array}{l}\text { DSM-IV } \\
\text { EDE }\end{array}$ & AN & PSWQ \\
\hline $\begin{array}{l}\text { Zarychta et al. } \\
2017[67]\end{array}$ & cross-sectional & $\begin{array}{c}\text { general } \\
\text { population: } 1260\end{array}$ & $16.38 \pm 0.80$ & $\begin{array}{l}\text { F: } 41.7 \% \\
\quad(525)\end{array}$ & MBSRQ & - & MBSRQ \\
\hline $\begin{array}{l}\text { Crino et al. } \\
2019 \text { [68] }\end{array}$ & case-control & $\begin{array}{c}\text { cases: } 90 \\
\text { controls: } 97\end{array}$ & $\begin{array}{c}25.23 \pm 8.33 \text { vs. } \\
20.63 \pm 6.37\end{array}$ & $\begin{array}{c}\text { F: } 100 \% \\
(187)\end{array}$ & DSM-5 & $\begin{array}{l}\text { AN BN BED } \\
\text { No-ED }\end{array}$ & TCQ \\
\hline $\begin{array}{l}\text { Hartmann } \\
\text { et al. } 2019 \text { [69] }\end{array}$ & cross-sectional & cases: 95 & $\begin{array}{l}\text { AN: } 23.64 \pm 0.62 \\
\text { BN: } 26.09 \pm 1.17\end{array}$ & $\begin{array}{c}\text { F: } 98 \%(49) \\
\text { F: } \\
97.78 \%(44)\end{array}$ & EDE-Q & $\begin{array}{l}\mathrm{AN} \\
\mathrm{BN}\end{array}$ & $\begin{array}{l}\text { Self-constructed } \\
\text { worry item }\end{array}$ \\
\hline
\end{tabular}

Note: AN: Anorexia Nervosa; BN: Bulimia Nervosa; BED: Binge Eating Disorder; CG: Control group; DSM-IV: Diagnostic and Statistical Manual of Mental Disorders; EAT: Eating Attitudes Test; EDE: Eating Disorder Examination; FOBES: Functional assessment of binge eating; PSWQ: Penn State Worry Questionnaire; CITQ: Control of Intrusive Thoughts Questionnaire; MBSRQ: The Multidimensional Body-Self Relations Questionnaire. 
Table 2. Summary of the demographic characteristics of the studies assessed the relationship between rumination and eating behavior.

\begin{tabular}{|c|c|c|c|c|c|c|c|}
\hline Source & $\begin{array}{l}\text { Study } \\
\text { Design }\end{array}$ & Sample-Size & $\begin{array}{l}\text { Age (Years) } \\
\text { Mean } \pm \text { SD }\end{array}$ & Sex \% $(n)$ & $\begin{array}{c}\text { Diagnostic } \\
\text { Tool } \\
\text { Eating } \\
\text { Measure }\end{array}$ & $\begin{array}{l}\text { Sub-Types of } \\
\text { ED Symptoms }\end{array}$ & $\begin{array}{c}\text { Rumination } \\
\text { Measure }\end{array}$ \\
\hline $\begin{array}{l}\text { González et al. } \\
2017 \text { [57] }\end{array}$ & $\begin{array}{l}\text { cross- } \\
\text { sectional }\end{array}$ & $\begin{array}{c}\text { general } \\
\text { population: } \\
176\end{array}$ & $31.2 \pm 13.3$ & F: 67\% (118) & EAT-26 & $\begin{array}{l}\mathrm{AN} \\
\mathrm{BN}\end{array}$ & RRS \\
\hline $\begin{array}{c}\text { Naumann et al. } \\
2015 \text { [59] }\end{array}$ & $\begin{array}{l}\text { experiment } \\
\text { case-control }\end{array}$ & $\begin{array}{c}\text { cases: } 36 \\
\text { controls: } 19\end{array}$ & $\begin{array}{l}\text { AN: } 24.94 \pm 8.92 \\
\text { BN: } 23.28 \pm 6.37 \\
\text { CG: } 23.32 \pm 8.02\end{array}$ & $\begin{array}{l}F: 100 \% \\
(111)\end{array}$ & $\begin{array}{l}\text { DSM-IV-TR } \\
\text { EDE }\end{array}$ & $\begin{array}{l}\mathrm{AN} \\
\mathrm{BN}\end{array}$ & $\begin{array}{c}\text { Rumination } \\
\text { experimentally } \\
\text { induced by } \\
\text { Nolen- } \\
\text { Hoeksema and } \\
\text { Morrow's task } \\
\text { (1993) } \\
\text { RSQ }\end{array}$ \\
\hline $\begin{array}{c}\text { Troop and } \\
\text { Treasure } 1997 \\
{[61]}\end{array}$ & case-control & $\begin{array}{c}\text { cases: } 21 \\
\text { controls: } 15\end{array}$ & $\begin{array}{c}\text { AN: } 23.3 \pm 5 \\
\text { BN: } 25.4 \pm 10.8 \\
\text { CG: } 29.5 \pm 9\end{array}$ & F: $100 \%(36)$ & ICD-10 & $\begin{array}{l}\mathrm{AN} \\
\mathrm{BN}\end{array}$ & $\begin{array}{l}\text { Coping } \\
\text { Strategies } \\
\text { Interview }\end{array}$ \\
\hline \multirow[t]{2}{*}{$\begin{array}{l}\text { Cowdrey and } \\
\text { Park } 2012 \text { [62] }\end{array}$} & \multirow[t]{2}{*}{$\begin{array}{l}\text { cross- } \\
\text { sectional }\end{array}$} & $\begin{array}{c}\text { general } \\
\text { population: } \\
228\end{array}$ & $24.03 \pm 7.62$ & \multirow[t]{2}{*}{$\begin{array}{c}\mathrm{F}: 100 \% \text { vs. } \\
100 \%\end{array}$} & \multirow[t]{2}{*}{ EDE-Q } & \multirow[t]{2}{*}{$\begin{array}{c}\text { AN and Eating } \\
\text { pathology }\end{array}$} & \multirow[t]{2}{*}{ RRS-ED } \\
\hline & & AN: 42 & $24 \pm 8.31$ & & & & \\
\hline \multirow{2}{*}{$\begin{array}{c}\text { Rawal et al. } \\
2010 \text { [63] }\end{array}$} & $\begin{array}{l}\text { Study } 1 \\
\text { cross- } \\
\text { sectional }\end{array}$ & students: 177 & $22.39 \pm 5.13$ & $\begin{array}{l}\text { F: } 68.92 \% \\
\quad(122)\end{array}$ & $\begin{array}{l}\text { DSM-IV } \\
\text { EDE-Q }\end{array}$ & \multirow{2}{*}{$\mathrm{AN}$} & \multirow{2}{*}{$\begin{array}{c}\text { Study } 1 \text { \& 2: } \\
\text { RRS }\end{array}$} \\
\hline & $\begin{array}{c}\text { Study } 2 \\
\text { case-control }\end{array}$ & $\begin{array}{l}\text { cases: } 13 \\
\text { controls: } 13\end{array}$ & $\begin{array}{c}26.38 \pm 8.77 \text { vs. } \\
25.77 \pm 4.85\end{array}$ & $\begin{array}{c}\mathrm{F}: 100 \% \text { vs. } \\
100 \%\end{array}$ & $\begin{array}{l}\text { MINI } \\
\text { EDE }\end{array}$ & & \\
\hline \multirow{2}{*}{$\begin{array}{c}\text { Wang and } \\
\text { Borders } 2018 \\
{[64]}\end{array}$} & $\begin{array}{l}\text { Study } 1 \\
\text { cross- } \\
\text { sectional }\end{array}$ & $\begin{array}{l}\text { undergraduate } \\
\text { students: } 126\end{array}$ & $19.7 \pm 1.10$ & F: 84\% (106) & \multirow{2}{*}{ EDE-Q } & \multirow{2}{*}{$\begin{array}{c}\text { Eating } \\
\text { pathology }\end{array}$} & \multirow{2}{*}{ RRS } \\
\hline & $\begin{array}{l}\text { Study } 2 \\
\text { cross- } \\
\text { sectional }\end{array}$ & cases: 85 & $24.57 \pm 9.95$ & F: 87.1\% (74) & & & \\
\hline \multirow{2}{*}{$\begin{array}{c}\text { Mason and } \\
\text { Lewis } 2017 \text { [65] }\end{array}$} & \multirow{2}{*}{$\begin{array}{l}\text { cross- } \\
\text { sectional }\end{array}$} & $\begin{array}{c}\text { general } \\
\text { population } \\
\text { Caucasian: } \\
100\end{array}$ & $20.14 \pm 1.82$ & \multirow[t]{2}{*}{$\mathrm{F}=100 \%$} & \multirow{2}{*}{$\begin{array}{c}\text { DSM-5 } \\
\text { (binge eating } \\
\text { episode) }\end{array}$} & \multirow[t]{2}{*}{-} & \multirow[t]{2}{*}{ RSQ } \\
\hline & & $\begin{array}{l}\text { African- } \\
\text { America: } \\
\quad 84\end{array}$ & $19.75 \pm 1.86$ & & & & \\
\hline $\begin{array}{l}\text { Startup et al. } \\
2013 \text { [66] }\end{array}$ & $\begin{array}{l}\text { cross- } \\
\text { sectional }\end{array}$ & cases: 62 & $26.6 \pm 7.8$ & $\begin{array}{l}F: 93.5 \% \\
\quad(58)\end{array}$ & $\begin{array}{l}\text { DSM-IV } \\
\text { EDE }\end{array}$ & $\mathrm{AN}$ & CERTS \\
\hline $\begin{array}{l}\text { Connolly et al. } \\
2007 \text { [70] }\end{array}$ & $\begin{array}{l}\text { cross- } \\
\text { sectional }\end{array}$ & $\begin{array}{c}\text { general } \\
\text { population: } \\
140\end{array}$ & $19.5 \pm 2.57$ & F 100\% (140) & $\begin{array}{c}\text { BES } \\
\text { EDE-Q }\end{array}$ & BED & BARQ \\
\hline $\begin{array}{c}\text { Nolen- } \\
\text { Hoeksema } \\
\text { et al. } 2007 \text { [71] }\end{array}$ & $\begin{array}{l}\text { cross- } \\
\text { sectional }\end{array}$ & $\begin{array}{c}\text { general } \\
\text { population: } \\
496\end{array}$ & $13.5 \pm 0.67$ & $\mathrm{~F}=100 \%$ & $\begin{array}{l}\text { DSM-IV } \\
\text { EDE }\end{array}$ & $\mathrm{BN}$ & RSQ \\
\hline $\begin{array}{c}\text { Harrell et al. } \\
2008 \text { [72] }\end{array}$ & $\begin{array}{l}\text { cross- } \\
\text { sectional }\end{array}$ & $\begin{array}{l}\text { general } \\
\text { population: } \\
329\end{array}$ & 19.31 & $\mathrm{~F}=100 \%$ & $\begin{array}{l}\text { Dieting and } \\
\text { Bingeing } \\
\text { Severity Scale }\end{array}$ & - & AFCI \\
\hline $\begin{array}{l}\text { Selby et al. } \\
2008 \text { [73] }\end{array}$ & $\begin{array}{l}\text { cross- } \\
\text { sectional }\end{array}$ & $\begin{array}{l}\text { general } \\
\text { population: } \\
200\end{array}$ & $18.6 \pm 2.36$ & F: 68.5\% (137) & EDI & $\mathrm{BN}$ & CERQ \\
\hline $\begin{array}{l}\text { Aldao and } \\
\text { Nolen- } \\
\text { Hoeksema } \\
2010 \text { [74] }\end{array}$ & $\begin{array}{l}\text { cross- } \\
\text { sectional }\end{array}$ & $\begin{array}{l}\text { undergraduate } \\
\text { students: } 252\end{array}$ & $18.44 \pm 0.66$ & F: $55.6 \%(140)$ & EDE-Q & - & RRS \\
\hline $\begin{array}{c}\text { Holm-Denoma } \\
\text { and Hankin } \\
2010[75]\end{array}$ & $\begin{array}{l}\text { cross- } \\
\text { sectional }\end{array}$ & $\begin{array}{c}\text { general } \\
\text { population: } \\
191\end{array}$ & $14.5 \pm 1.4$ & $\mathrm{~F}=100 \%$ & EDDS & $\mathrm{BN}$ & CRSQ \\
\hline
\end{tabular}


Table 2. Cont.

\begin{tabular}{|c|c|c|c|c|c|c|c|}
\hline Source & $\begin{array}{l}\text { Study } \\
\text { Design }\end{array}$ & Sample-Size & $\begin{array}{l}\text { Age (Years) } \\
\text { Mean } \pm \text { SD }\end{array}$ & Sex \% $(n)$ & $\begin{array}{l}\text { Diagnostic } \\
\text { Tool } \\
\text { Eating } \\
\text { Measure }\end{array}$ & $\begin{array}{l}\text { Sub-Types of } \\
\text { ED Symptoms }\end{array}$ & $\begin{array}{l}\text { Rumination } \\
\text { Measure }\end{array}$ \\
\hline $\begin{array}{c}\text { Verplanken } \\
\text { and Tangelder } \\
2011 \text { [76] }\end{array}$ & $\begin{array}{l}\text { cross- } \\
\text { sectional }\end{array}$ & students: 303 & $24 \pm 4$ & $\begin{array}{l}\text { F: } 50.16 \% \\
\quad(152)\end{array}$ & EDS-5 & - & $\begin{array}{l}\text { Negative Body } \\
\text { Image } \\
\text { Thinking }\end{array}$ \\
\hline $\begin{array}{l}\text { Gordon et al. } \\
2012[77]\end{array}$ & $\begin{array}{l}\text { cross- } \\
\text { sectional }\end{array}$ & $\begin{array}{l}\text { general } \\
\text { population: } \\
780\end{array}$ & $19.27 \pm 2.12$ & F: 65.7\% (512) & BES & BED & RRS \\
\hline $\begin{array}{l}\text { Kelly et al. } \\
2012 \text { [78] }\end{array}$ & $\begin{array}{l}\text { cross- } \\
\text { sectional }\end{array}$ & $\begin{array}{l}\text { general } \\
\text { population: } \\
419\end{array}$ & $18.95 \pm 1.33$ & $\mathrm{~F}=100 \%$ & EDE-Q & BED & CERQ \\
\hline $\begin{array}{c}\text { Hilt et al. } 2013 \\
\text { [79] }\end{array}$ & $\begin{array}{c}\text { cross- } \\
\text { sectional }\end{array}$ & $\begin{array}{l}\text { general } \\
\text { population: } \\
101\end{array}$ & $12.7 \pm 1.14$ & $\mathrm{~F}=100 \%$ & $\begin{array}{l}\text { Children's } \\
\text { Eating } \\
\text { Attitudes Test } \\
\text { ChEAT }\end{array}$ & $\begin{array}{l}\text { Eating } \\
\text { pathology }\end{array}$ & CRSQ \\
\hline $\begin{array}{c}\text { Svaldi and } \\
\text { Naumann } 2014 \\
\text { [80] } \\
\end{array}$ & $\begin{array}{l}\text { cross- } \\
\text { sectional }\end{array}$ & cases: 30 & 46.33 & $\mathrm{~F}=100 \%$ & $\begin{array}{l}\text { DSM-IV-TR } \\
\text { EDE }\end{array}$ & BED & PTQ \\
\hline $\begin{array}{l}\text { Mason and } \\
\text { Lewis } 2015 \text { [81] }\end{array}$ & $\begin{array}{l}\text { cross- } \\
\text { sectional }\end{array}$ & $\begin{array}{l}\text { general } \\
\text { population: } \\
164\end{array}$ & - & $F=100 \%$ & BES & BED & CERQ \\
\hline $\begin{array}{l}\text { Breithaupt } \\
\text { et al. } 2016 \text { [82] }\end{array}$ & $\begin{array}{l}\text { cross- } \\
\text { sectional }\end{array}$ & $\begin{array}{l}\text { general } \\
\text { population: } \\
353 \\
\end{array}$ & $21.93 \pm 5.78$ & $\mathrm{~F}=85 \%(300)$ & EAT-26 & $\mathrm{BN}$ & RRS \\
\hline $\begin{array}{l}\text { Jungmann et al. } \\
2016 \text { [83] }\end{array}$ & $\begin{array}{l}\text { cross- } \\
\text { sectional }\end{array}$ & $\begin{array}{l}\text { general } \\
\text { population: } \\
414\end{array}$ & $47.2 \pm 16.7$ & F: 54\% (223) & EDI-2 & $\mathrm{BN}$ & RSQ \\
\hline $\begin{array}{l}\text { Maraldo et al. } \\
2016 \text { [84] }\end{array}$ & $\begin{array}{l}\text { cross- } \\
\text { sectional }\end{array}$ & $\begin{array}{l}\text { community } \\
\text { participants: } \\
313 \\
\text { students: } 296\end{array}$ & $\begin{array}{l}34.74 \pm 11.36 \text { vs. } \\
19.44 \pm 1.75\end{array}$ & $\mathrm{~F}=100 \%$ & EDE-Q & $\begin{array}{l}\text { Eating } \\
\text { pathology }\end{array}$ & RRS \\
\hline $\begin{array}{l}\text { Naumann et al. } \\
2016 \text { [85] }\end{array}$ & case-control & $\begin{array}{l}\text { cases: AN: } \\
42 \text { BN: } 40 \\
\text { controls: } 41\end{array}$ & $\begin{array}{c}\text { AN: } 25.71 \pm 10.65 \\
\text { BN: } 25.78 \pm 8.49 \\
\text { CG: } 25.61 \pm 10.30\end{array}$ & $\mathrm{~F}=100 \%$ & $\begin{array}{l}\text { DSM-IV } \\
\text { EDE }\end{array}$ & $\begin{array}{l}\mathrm{AN} \\
\mathrm{BN}\end{array}$ & $\begin{array}{c}\text { Self- } \\
\text { constructed } \\
\text { Visual Analog } \\
\text { Scales }\end{array}$ \\
\hline $\begin{array}{l}\text { Seidel et al. } \\
2016 \text { [86] }\end{array}$ & case-control & $\begin{array}{c}\text { cases: } 37 \\
\text { controls: } 33\end{array}$ & $\begin{array}{l}\text { AN: } 16.40 \pm 2.33 \\
\text { CG: } 16.51 \pm 3.79\end{array}$ & $\mathrm{~F}=100 \%$ & $\begin{array}{l}\text { DSM-IV } \\
\text { EDI-2 }\end{array}$ & AN & PTQ \\
\hline $\begin{array}{l}\text { Opwis et al. } \\
2017 \text { [87] }\end{array}$ & $\begin{array}{l}\text { cross- } \\
\text { sectional }\end{array}$ & $\begin{array}{l}\text { general } \\
\text { population: } \\
295\end{array}$ & $\begin{array}{c}\text { F: } 30.23 \pm 8.94 \\
\text { M: } 30.76 \pm 9.14\end{array}$ & F: 69\% (205) & EDE-Q & $\begin{array}{l}\text { Eating } \\
\text { pathology }\end{array}$ & RS-8 \\
\hline $\begin{array}{c}\text { Wang and } \\
\text { Borders } 2017 \\
\text { [88] }\end{array}$ & $\begin{array}{l}\text { cross- } \\
\text { sectional }\end{array}$ & $\begin{array}{l}\text { general } \\
\text { population: } \\
116\end{array}$ & $24.8 \pm 5.35$ & M: 59.5\% (69) & EAT-26 & $\begin{array}{l}\text { Eating } \\
\text { pathology }\end{array}$ & $\begin{array}{l}\text { Items modified } \\
\text { from the } \\
\text { Rumination } \\
\text { About } \\
\text { Interpersonal } \\
\text { Offences Scale }\end{array}$ \\
\hline $\begin{array}{l}\text { Wang et al. } \\
2017 \text { [89] }\end{array}$ & $\begin{array}{c}\text { cross- } \\
\text { sectional }\end{array}$ & cases: 237 & $47.9 \pm 10$ & F: 70\% (167) & $\begin{array}{l}\text { DSM-IV-TR } \\
\text { EDE }\end{array}$ & BED & RRS \\
\hline $\begin{array}{l}\text { Dondzillo et al. } \\
\quad 2018 \text { [90] }\end{array}$ & $\begin{array}{l}\text { cross- } \\
\text { sectional }\end{array}$ & $\begin{array}{l}\text { general } \\
\text { population: } \\
73\end{array}$ & $18.59 \pm 1.28$ & $\mathrm{~F}=100 \%$ & DEBQ & AN & RRS-ED \\
\hline $\begin{array}{l}\text { Van Durme } \\
\text { et al. } 2018 \text { [91] }\end{array}$ & $\begin{array}{l}\text { cross- } \\
\text { sectional }\end{array}$ & $\begin{array}{l}\text { general } \\
\text { population: } \\
397\end{array}$ & 14.02 & F: $62.7 \%(249)$ & EDI-II & $\mathrm{BN}$ & FEEL-KJ \\
\hline $\begin{array}{l}\text { Birmachu et al. } \\
\quad 2019 \text { [92] }\end{array}$ & $\begin{array}{l}\text { cross- } \\
\text { sectional }\end{array}$ & $\begin{array}{l}\text { general } \\
\text { population: } \\
300\end{array}$ & $22.99 \pm 6.91$ & F: 63.6\% (190) & EDE-Q & $\begin{array}{l}\text { Eating } \\
\text { pathology }\end{array}$ & RRQ \\
\hline
\end{tabular}


Table 2. Cont.

\begin{tabular}{|c|c|c|c|c|c|c|c|}
\hline Source & $\begin{array}{l}\text { Study } \\
\text { Design }\end{array}$ & Sample-Size & $\begin{array}{l}\text { Age (Years) } \\
\text { Mean } \pm \text { SD }\end{array}$ & $\operatorname{Sex} \%(n)$ & $\begin{array}{c}\text { Diagnostic } \\
\text { Tool } \\
\text { Eating } \\
\text { Measure }\end{array}$ & $\begin{array}{l}\text { Sub-Types of } \\
\text { ED Symptoms }\end{array}$ & $\begin{array}{c}\text { Rumination } \\
\text { Measure }\end{array}$ \\
\hline $\begin{array}{l}\text { Fresnics et al. } \\
2019 \text { [93] }\end{array}$ & $\begin{array}{l}\text { cross- } \\
\text { sectional }\end{array}$ & $\begin{array}{c}\text { general } \\
\text { population: } \\
190\end{array}$ & $19.3 \pm 1.10$ & F: $84 \%(160)$ & EDE-Q & $\begin{array}{c}\text { Eating } \\
\text { pathology }\end{array}$ & RRS \\
\hline $\begin{array}{l}\text { Hernando et al. } \\
2019 \text { [94] }\end{array}$ & case-control & $\begin{array}{c}\text { cases: } 25 \\
\text { controls: } 25\end{array}$ & $\begin{array}{c}16.6 \pm 2.24 \text { vs. } \\
19.08 \pm 0.64\end{array}$ & $\mathrm{~F}=100 \%$ & - & $\begin{array}{l}\text { AN, BN, } \\
\text { OSFED }\end{array}$ & RSQ \\
\hline $\begin{array}{l}\text { Smith et al. } \\
2019 \text { [95] }\end{array}$ & $\begin{array}{l}\text { cross- } \\
\text { sectional }\end{array}$ & $\begin{array}{l}\text { undergraduate } \\
\text { students: } 263\end{array}$ & $20.3 \pm 3.68$ & F: 74.9\% (197) & EDDS & $\begin{array}{c}\text { Eating } \\
\text { pathology }\end{array}$ & RRS \\
\hline $\begin{array}{c}\text { Branley-Bell } \\
\text { and Talbot } \\
2020[96]\end{array}$ & $\begin{array}{l}\text { cross- } \\
\text { sectional }\end{array}$ & $\begin{array}{c}\text { general } \\
\text { population: } \\
129\end{array}$ & $9.27 \pm 8.99$ & F: $93.8 \%(121)$ & $\begin{array}{l}\text { Self-reported } \\
\text { ED }\end{array}$ & $\begin{array}{c}\text { Eating } \\
\text { pathology }\end{array}$ & RRS-ED \\
\hline
\end{tabular}

Note: AN: Anorexia Nervosa; BN: Bulimia Nervosa; CG: control group; OSFED: Other Specified Feeding or Eating Disorders; ICD: International Classification of Diseases; DSM: Diagnostic and Statistical Manual of Mental Disorders; EAT-26: Eating Attitudes: Test EDE: Eating Disorder Examination; EDDS: Eating Disorder Diagnostic Scale; EDI-2: Eating Disorder Inventory; RSQ: Response Styles Questionnaire; BES: Binge Eating Scale; EDE-Q: Eating Disorders Examination Questionnaire; EDS-5: Eating Disturbance Scale; BARQ: Behavioural Anger Response Questionnaire; RRS: Ruminative Response Scale; RRS-ED: Ruminative Response Scale for Eating Disorders; MINI: Mini International Neuropsychiatric Interview; AFCI: Adult Emotion-Focused Coping Inventory; ChEAT: Children's Eating Attitudes Test; CRSQ: Children's Response Style Questionnaire; CERQ: Cognitive Emotion Regulation Questionnaire; EDE-Q: Eating Disorder Examination Questionnaire; RS-8: Rumination-Suppression-8 Scale; CERTS: Cambridge Exeter Repetitive Thought Scale; DEBQ: Dutch Eating Behaviour Questionnaire; FEEL-KJ: Fragebogen zur Erhebung der Emotionsregulation bei Kindern und Jugendlich; RRQ: Rumination-reflection Questionnaire.

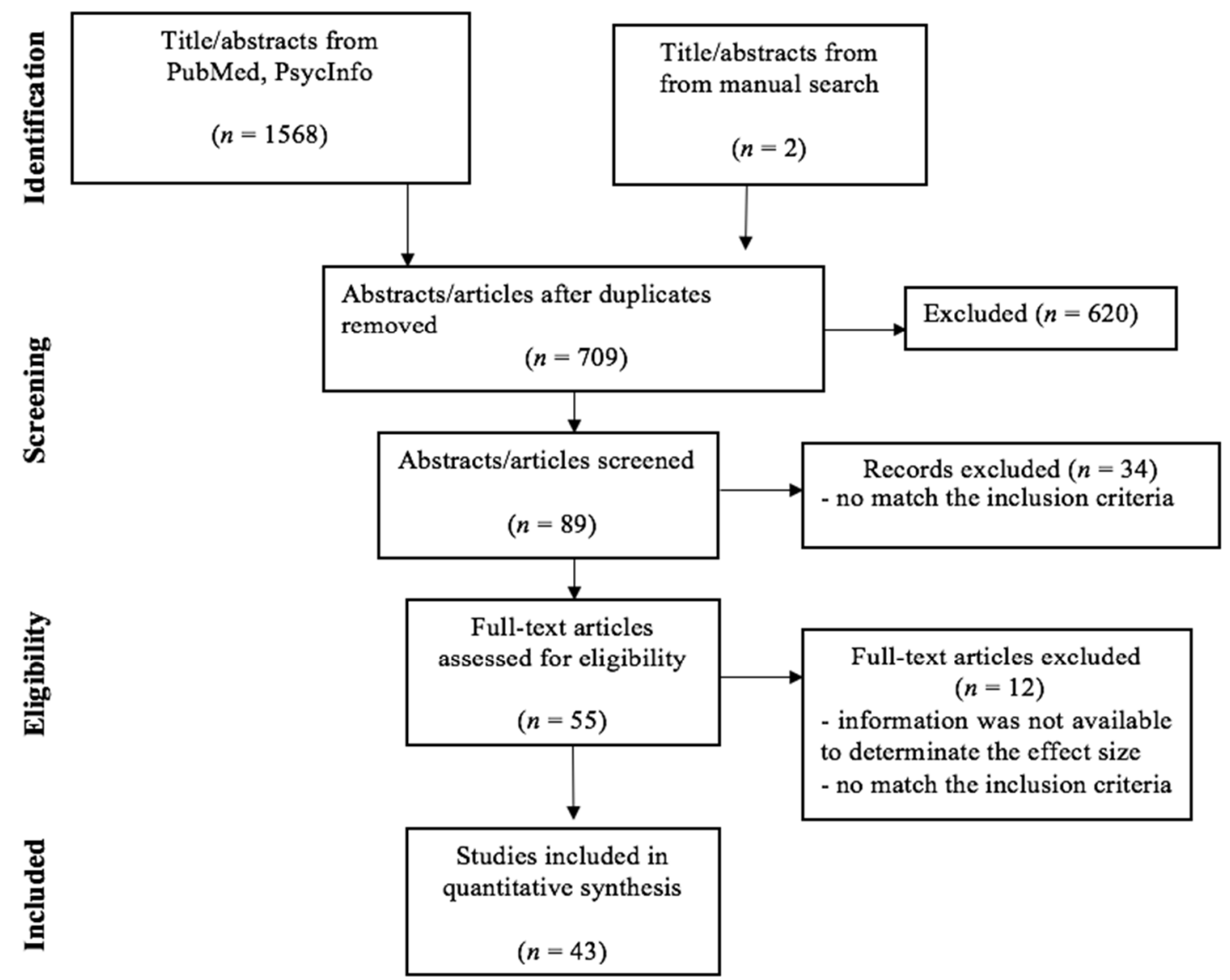

Figure 1. Identification of independent studies for inclusion in the meta-analysis (flow chart). Forty-three studies of 57 cohorts, because some studies were considered more than once. Search Strategy: Limits: English; Search terms included: worry/rumination/brooding/repetitive thinking combined using Boolean "AND" operator with eating disorder/anorexia/bulimia/binge eating disorder. 


\subsection{Study Quality}

None of the studies fulfilled all the Newcastle-Ottawa quality criteria. In twelve casecontrol studies, six studies scored $7 / 10$, three studies scored equal to 6 and three studies scored 5/10. In thirty-three cross-sectional studies, three studies scored 8/10, 26 studies scored equal to 7 , three study scored $6 / 10$ and one scored $5 / 10$. More details about the study quality for the case-control and cohort studies are reported in the Supplementary Materials in Tables S1 and S2, respectively.

\subsection{RT and Eating Problems}

The 57 entries selected showed an average ES of 0.85 (SE: 0.07, 95\% CI: 0.72-0.98; Test of Null (2-Tail): $z$-value $=12.61, p<0.001, k=57$ ), which indicated a large positive association between eating problems and RNT (Figure 2). The ES is a global measure and has been calculated in all the studies included in the quantitative analysis. Duval and Tweedie's trim-and-fill procedure indicated only five missing studies (adjusted ES $g=0.90,95 \%$ CI: $0.75-1.06)$, with a classic fail-safe number estimated at 6897 . Since we found heterogeneity among the studies $\left(\mathrm{Q}=559.79, p<0.001, \mathrm{I}^{2}=90.000\right)$, we conducted further analyses by testing the roles of the possible moderators.

\section{Meta Analysis}

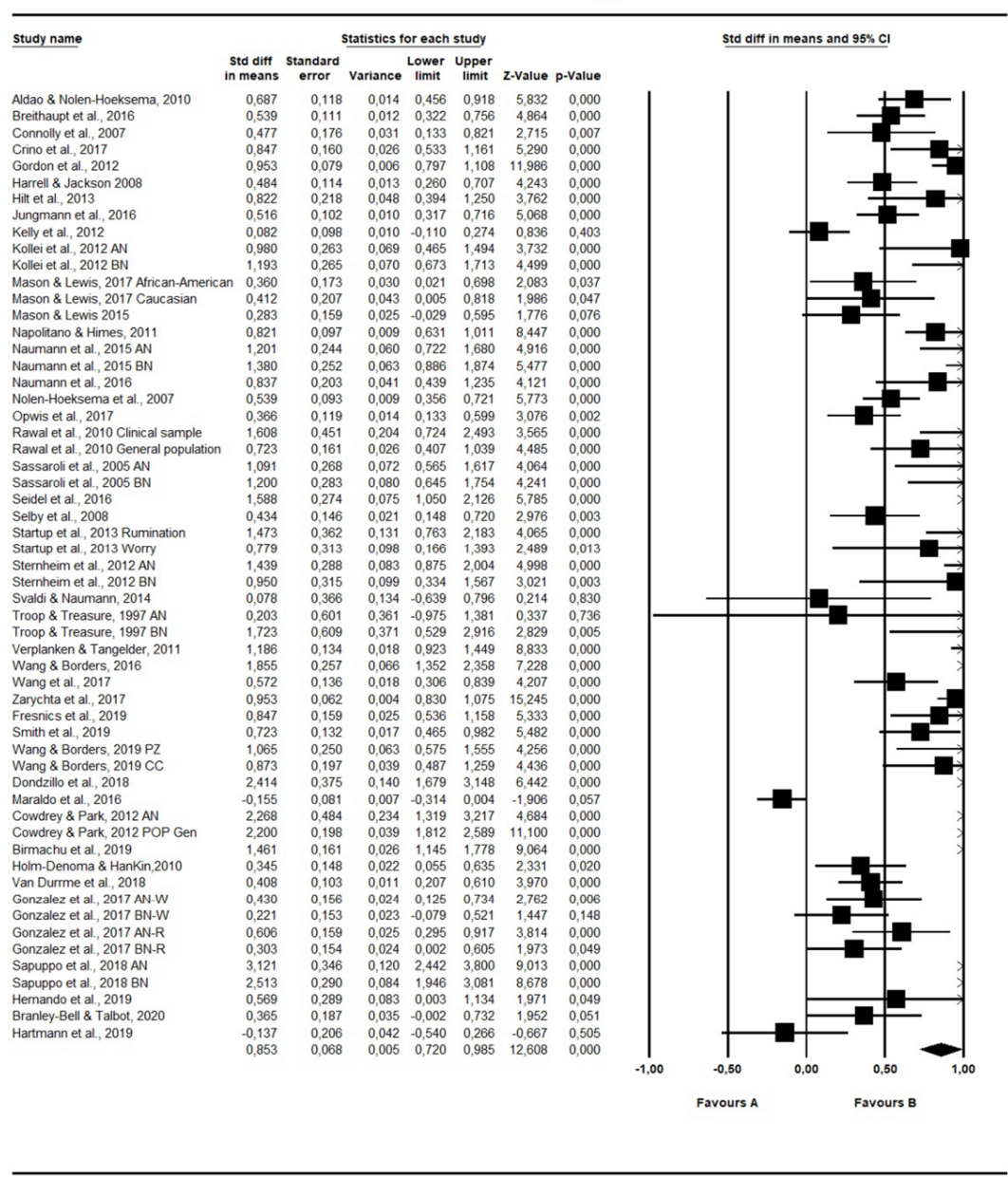

\section{Meta Analysis}

Figure 2. Forest plots for repetitive thinking and eating behavior. Box indicates effect sizes (ESs), while error bars represent 95\% confidence intervals (CIs). The rumble represents the average ES. Note: Favors A = negative association between repetitive thinking and eating disorders. Favors $\mathrm{B}=$ positive association between repetitive thinking and eating disorders. The rumble represents the average ES. 
Moderator Analyses: Subtypes of ED Symptoms, Presence vs. Absence of Diagnosis of EDs, Worry-Rumination and Age

For each moderator, a separate model was tested. For more details, see Table 3 . The subgroup analysis revealed differences in ES for the types of ED symptoms $(\mathrm{Q}=11.65$, $d f=2, p=0.003$ ): AN $d=1.35$ (SE:0.20,95\% CI: 0.96-1.74; Test of Null (2-Tail): $z$-value $=7.76$, $p<0.001, k=14), \mathrm{BN} d=0.75$ (SE:0.11,95\% CI: 0.54-0.97; Test of Null (2-Tail): $z$-value $=6.80$, $p<0.001, k=14$ ); BED, $d=0.50$ (SE:0.15, 95\% CI: 0.20-0.80; Test of Null (2-Tail): $z$-value = 3.29, $p=0.001, k=7)$.

We also found differences in the ES between studies based on clinical samples vs. studies based on the healthy controls from the general population $(\mathrm{Q}=8.15, d f=1$, $p=0.004$ ): clinical samples ES: $d=1.14$ (SE: 0.13, 95\% CI: 0.87-1.40; Test of Null (2-Tail): $z$-value $=8.44, p<0.001, k=24)$; general population ES: $d=0.69$ (SE:0.08, 95\% CI: 0.54-0.85; Test of Null (2-Tail): $z$-value $=8.97, p<0.001, k=33$ ).

No differences emerged from the comparison between the studies on worry vs. studies on rumination $(\mathrm{Q}=2.45, \mathrm{df}=1, p=0.118)$ : worry $\mathrm{ES}: d=1.04$ (SE: 0.14, 95\% CI: 0.76-1.32, Test of Null 2-Tail: $z$-value $=7.28, p<0.001, k=15$ ); rumination ES: $d=0.79$ (SE: $0.08,95 \% \mathrm{CI}$ : 0.64-0.94, Test of Null 2-Tail: $z$-value $=10.36, p<0.001, k=42$ ).

Finally, there was not a significant effect of the variable "age" in shaping heterogeneity (age: $\beta$ point estimate $=-0.0108, \mathrm{SE}=0.009,95 \% \mathrm{CI}:-0.03-0.01, z$-value $=-1.12, p=0.26$, $k=55)$.

Table 3. Moderator analyses: subtypes of ED symptoms, presence vs. absence of diagnosis of EDs, worry-rumination and age.

\begin{tabular}{|c|c|c|c|c|c|}
\hline & ES & $\beta$ Point Estimate & SE & CI & $p$ \\
\hline Subtypes of ED symptoms & & & & & 0.003 \\
\hline $\mathrm{AN}$ & 1.35 & & 0.20 & $0.96-1.74$ & $<0.001$ \\
\hline $\mathrm{BN}$ & 0.75 & & 0.11 & $0.54-0.97$ & $<0.001$ \\
\hline BED & 0.50 & & 0.15 & $0.20-0.80$ & 0.001 \\
\hline Presence vs. absence of diagnosis of EDs & & & & & 0.004 \\
\hline Clinical samples & 1.14 & & 0.13 & $0.87-1.40$ & $<0.001$ \\
\hline Healthy controls from the general population & 0.69 & & 0.08 & $0.54-0.85$ & $<0.001$ \\
\hline Repetitive Negative Thinking & & & & & 0.118 \\
\hline Worry & 1.04 & & 0.14 & $0.76-1.32$ & $<0.001$ \\
\hline Rumination & 0.79 & & 0.08 & $0.64-0.94$ & $<0.001$ \\
\hline Age & & -0.0108 & 0.009 & $-0.03-0.01$ & 0.26 \\
\hline
\end{tabular}

Note: $\mathrm{ES}$ = Effect Size; SE = Standard Error; CI = Confidence Interval; AN = Anorexia Nervosa; BN = Bulimia Nervosa; BED: Binge Eating Disorder.

\section{Discussion}

To our knowledge, this is the first study on the relationship between EDs, worry and rumination based on a meta-analytic methodology. The main findings of the present meta-analysis indicate a significant association between RNT and EDs, given that: (a) RNT is highly associated with eating problems in both clinical and nonclinical samples (ESs range from 0.50 to 1.35) and (b) ED patients show higher levels of RNT than the general population. These findings are consistent with Smith and colleagues' findings [30] but differ from these given that both worry and rumination have been found to be associated with ED symptoms. Moreover, our findings suggest that the strength of the association is not influenced by the age of the participants.

These findings raise an important question: How does RNT play a role in ED symptoms, such as dieting or binge eating? It has been shown that negative beliefs and negative emotions might act as a trigger for RNT that, in turn, further maintains the experience of emotional distress [2,3,66,97-99]. Moreover, it is well-known that dieting and binge eating could be a coping strategy to tackle negative emotions $[29,99,100]$. Should the latter per- 
spective be taken, dieting could be construed as a strategy to cope with negative thoughts and /or emotions that act as a trigger of RNT. Binge eating could be a behavior aimed to reduce chronic stress due to RNT focused on dieting or independently from it. Even though clinical models, such as the Self-Regulatory Executive Function (S-REF) model [101], the Emotional Cascade Model (ECM) [73] or Fairburn's model [29], might partially support these assumptions, further studies are required to directly test these hypotheses.

The Self-Regulatory Executive Function model (S-REF model) [101] postulates that several maladaptive forms of coping, including repetitive negative thinking (desire thinking, rumination and worry), maintain psychological distress. These maladaptive forms of coping are termed the "Cognitive Attentional Syndrome" (CAS) [102], which is activated and maintained by metacognitive beliefs (i.e., information individuals hold about their own cognition and coping) [102]. CAS is problematic, because it causes negative cognitiveaffective states to remain in the consciousness rather than spontaneously decay, leading to failures to modify self-beliefs about control over the mind [103]. Given the association observed between RNT and ED symptoms, and the association between metacognitive beliefs and both EDs and behaviors [48], the S-REF model could explain the role of RNT in ED symptoms.

As postulated by the emotional cascade model [73], an event that triggers a negative emotion may lead to rumination about the event, increasing the intensity of the negative emotions. Furthermore, stress and negative emotional states may increase the level of rumination, which, in turn, may lead to an escalation (cascade) of negative feelings [104]. As a result, an individual may engage in eating behaviors as a coping strategy to tackle negative mood states. It may be assumed that worry and negative emotion are related to each other in the same way. There is mounting evidence that worry may maintain the experience of emotional distress [98].

As regards the Fairburn's "transdiagnostic" model of ED [29], it was proposed that binge eating could be triggered, among other factors, by adverse events and negative mood states. In turn, binge eating will tend to improve, albeit temporarily, a negative mood and serve as a distraction from negative thinking patterns. It can therefore be argued that an ED mindset characterized by cognitive processes such as worry and rumination exist, and this is activated in the presence of adverse events, leading to a negative mood and the maintenance of ED behaviors. These behaviors may serve to decrease negative emotions, interrupt worry and rumination and help manage (in the short-term) the adverse event. However, the manner in which worry, rumination and negative affectivity may interact in EDs remains unclear, with further studies required to disentangle this complex relationship.

The findings of the current meta-analysis suggest that RNT is associated with all subtypes of ED symptoms supporting a vision of RNT as a transdiagnostic process [2]; nevertheless, some differences may be identified in the strength of the association between RNT and the subtypes of ED symptoms: based on the ES values, the association between RNT and the subtypes of ED symptoms might be stronger in AN than in BN and BED. Taking as a framework the S-REF model [101], it could be hypothesized that there are differences among the ED symptom subtypes in maladaptive metacognitive beliefs that activate and maintain maladaptive forms of coping such as RNT; as highlighted in a recent systematic review [48], maladaptive metacognitive beliefs appear to be stronger in AN than in other ED subtypes. Furthermore, the moderate ES for BED could suggest that BED symptoms could be more closely related with a different form of RNT process such as desire thinking $[78,105]$ rather than worry and rumination, albeit the scarce number of published studies on BED suggests caution in this interpretation.

Furthermore, our data, differently from a previous meta-analysis that exclusively focused on rumination [30], suggests that there is no difference between worry and rumination in the relationship with eating problems. This suggests, in line with the construct of RNT [2,3], that both processes are implicated in eating problems. Notwithstanding this observation, the additional data on worry may be in line with some evidence suggesting 
that, beyond having a common characteristic in repetitive thought, rumination and worry may have differential effects on the severity of mental illness [106,107]. Compared to rumination, worry appears to be a more influential cognitive vulnerability factor in predicting the increasing symptoms over time [106]. Focusing on the ED symptoms, negative thoughts about the weight and shape could activate worrying about eating and weight gain as a control strategy and associated negative beliefs and emotions; moreover, the worry about food may be a distraction from the preoccupations regarding self-esteem and interpersonal relations [32]. However, people with ED symptoms might also be worried about factors not strictly connected to the core features of EDs.

Finally, our data showed that age does not moderate the relationship between RNT and eating problems, leading to the hypothesis that the vicious circle among RNT, negative beliefs and emotions and eating behaviors could be independent from the passing of time. However, our data does not allow for further speculations on this issue.

A number of clinical and research implications rose from the findings of the current meta-analysis. Firstly, the assessment of RNT, in terms of worry and rumination, should not be overlooked during the anamnesis of a patient presenting with eating problems. Secondly, it could be important to inform patients presenting with eating problems that RNT is a disadvantageous mechanism that leads to worse clinical outcomes [108]. Thirdly, a treatment aimed to decrease the propensity to engage in RNT [108], such as Metacognitive Therapy [12] and Rumination-Based Therapy [109], should be considered as treatment options for EDs. Moreover, and based on the observed relationship between RNT and eating problems in the general population $(E S=0.69)$, early intervention in tackling RNT may help prevent more severe forms of problematic eating behaviour. Future research on EDs and eating problems could explore in depth the role of worry and rumination.

The value of this meta-analysis should be interpreted considering the strengths and limitations of the included studies. A significant strength is that the investigation of the relationship between worry, rumination and EDs was conducted across different subtypes of EDs, rather than in a specific ED, emphasizing the role of RNT in EDs. Some limitations should be also considered. The instruments used in the reviewed studies to evaluate the worry and rumination are not homogeneous: some studies used an instrument specific for ED (Ruminative Response Scale for Eating Disorders, RRS-ED); others used generic instruments (Ruminative Response Scale, RRS; Penn State Worry Questionnaire, PSWQ) or carried out an experiment. The majority of the studies were retrospective; hence, they are subject to a possible recall bias. Furthermore, the sample sizes were often small and composed only of female participants. Moderator analyses could be affected by this specific limitation. Thus, the analyses on moderators should be considered to be exploratory in nature. Moreover, focusing exclusively on published studies entails that the information about negative results is likely to be lost [110].

\section{Conclusions}

In conclusion, RNT represents a transdiagnostic phenomenon also involved in EDs. Future directions for research should include studies that: (1) explore in depth the worry and rumination in BED, since most studies have focused on AN and BN; (2) evaluate the relationship between RNT and EDs considering the possible confounder variables such as anxiety and depression [63,111]; (3) explore the relationship between RNT and negative emotion in EDs and (4) include longitudinal designs.

Supplementary Materials: The following are available online at https:/ /www.mdpi.com/article/10 .3390/jcm10112448/s1, Table S1: Quality Assessment for Case-Control Studies using the NewcastleOttawa Scale, Table S2: Quality Assessment for Cross Sectional Studies using the NewcastleOttawa Scale.

Author Contributions: Conceptualization, acquisition, interpretation of data S.P. and G.M.; formal analysis S.S. (Simona Scaini) and S.P.; writing-original draft preparation S.P., G.M., S.S. (Simona Scaini); writing-review and editing S.P., G.M, S.S. (Simona Scaini), W.S., M.M.S.; supervision 
G.C., S.S. (Sandra Sassaroli), G.M.R. All authors have read and agreed to the published version of the manuscript.

Funding: This research received no external funding.

Institutional Review Board Statement: Not applicable.

Informed Consent Statement: Not applicable.

Data Availability Statement: Not applicable.

Conflicts of Interest: The authors declare no conflict of interest.

\section{References}

1. Segerstrom, S.C.; Stanton, A.L.; Alden, L.E.; Shortridge, B.E. A Multidimensional Structure for Repetitive Thought: What's on Your Mind, and How, and How Much? J. Pers. Soc. Psychol. 2003, 85, 909. [CrossRef]

2. Ehring, T.; Watkins, E.R. Repetitive Negative Thinking as a Transdiagnostic Process. Int. J. Cogn. Ther. 2008, 1, 192-205. [CrossRef]

3. Watkins, E.R. Constructive and unconstructive repetitive thought. Psychol. Bull. 2008, 134, 163-206. [CrossRef] [PubMed]

4. Borkovec, T.; Robinson, E.; Pruzinsky, T.; DePree, J.A. Preliminary exploration of worry: Some characteristics and processes. Behav. Res. Ther. 1983, 21, 9-16. [CrossRef]

5. Nolen-Hoeksema, S.; Morrow, J. A prospective study of depression and posttraumatic stress symptoms after a natural disaster: The Lorma Prieta earthquake. J. Pers. Soc. Psychol. 1991, 65, 115-121. [CrossRef]

6. Papageorgiou, C. Worry and rumination: Styles of persistent negative thinking in anxiety and depression. In Worry and Its Psychological Disorder; Davey, G.C.L., Wells, A., Eds.; John Wiley: Chichester, UK, 2006; pp. 21-40.

7. Watkins, E. Appraisals and strategies associated with rumination and worry. Pers. Individ. Differ. 2004, 37, 679-694. [CrossRef]

8. Olatunji, B.O.; Naragon-Gainey, K.; Wolitzky-Taylor, K.B. Specificity of Rumination in Anxiety and Depression: A Multimodal Meta-Analysis. Clin. Psychol. 2013, 20, 225-257. [CrossRef]

9. Chorpita, B.F.; Tracey, S.A.; Brown, T.A.; Collica, T.J.; Barlow, D.H. Assessment of worry in children and adolescents: An adaptation of the Penn State Worry Questionnaire. Behav. Res. Ther. 1997, 35, 569-581. [CrossRef]

10. Muris, P.; Meesters, C.; Merckelbach, H.; Sermon, A.; Zwakhalen, S. Worry in Normal Children. J. Am. Acad. Child Psychiatry 1998, 37, 703-710. [CrossRef]

11. Perrin, S.; Last, C.G. Worrisome thoughts in children referred for anxiety disorder. J. Clin. Child Adolesc. Psychol. 1997, 26, 181-189. [CrossRef] [PubMed]

12. Wells, A. Metacognitive Therapy for Anxiety and Depression; The Guilford Press: New York, NY, USA, 2009.

13. Andrews, V.H.; Borkovec, T. The differential effects of inductions of worry, somatic anxiety, and depression on emotional experience. J. Behav. Ther. Exp. Psychiatry 1988, 19, 21-26. [CrossRef]

14. Chelminski, I.; Zimmerman, M. Pathological worry in depressed and anxious patients. J. Anxiety Disord. 2003, 17, 533-546. [CrossRef]

15. Starcevic, V. Pathological worry in major depression: A preliminary report. Behav. Res. Ther. 1995, 33, 55-56. [CrossRef]

16. Calmes, C.A.; Roberts, J.E. Repetitive Thought and Emotional Distress: Rumination and Worry as Prospective Predictors of Depressive and Anxious Symptomatology. Cogn. Ther. Res. 2007, 31, 343-356. [CrossRef]

17. Clark, D.M.; Wells, A. A cognitive model of social phobia. In Social Phobia: Diagnosis, Assessment and Treatment; Heimberg, R., Liebowitz, M., Hope, D.A., Schneier, F.R., Eds.; Guilford Press: New York, NY, USA, 1995.

18. Fresco, D.M.; Frankel, A.N.; Mennin, D.S.; Turk, C.L.; Heimberg, R.G. Distinct and Overlapping Features of Rumination and Worry: The Relationship of Cognitive Production to Negative Affective States. Cogn. Ther. Res. 2002, 26, 179-188. [CrossRef]

19. Harrington, J.A.; Blankenship, V. Ruminative Thoughts and Their Relation to Depression and Anxiety. J. Appl. Soc. Psychol. 2002, 32, 465-485. [CrossRef]

20. Kashdan, T.B.; Roberts, J.E. Social anxiety, depressive symptoms, and post-event rumination: Affective consequences and social contextual influences. J. Anxiety Disord. 2007, 21, 284-301. [CrossRef]

21. McLaughlin, K.A.; Nolen-Hoeksema, S. Rumination as a transdiagnostic factor in depression and anxiety. Behav. Res. Ther. 2011, 49, 186-193. [CrossRef]

22. Mellings, T.M.; Alden, L.E. Cognitive processes in social anxiety: The effects of self-focus, rumination and anticipatory processing. Behav. Res. Ther. 2000, 38, 243-257. [CrossRef]

23. de Silveira, M.É., Jr.; Kauer-Sant'Anna, M. Rumination in bipolar disorder: A systematic review. Rev. Bras. Psiquiatr. 2015, 37, 256-263. [CrossRef]

24. Vassilopoulos, S.P. Social anxiety and ruminative self-focus. J. Anxiety Disord. 2008, 22, 860-867. [CrossRef] [PubMed]

25. Caselli, G.; Ferretti, C.; Leoni, M.; Rebecchi, D.; Rovetto, F.; Spada, M.M. Rumination as a predictor of drinking behaviour in alcohol abusers: A prospective study. Addiction 2010, 105, 1041-1048. [CrossRef] [PubMed]

26. Devynck, F.; Kornacka, M.; Sgard, F.; Douilliez, C. Repetitive Thinking in Alcohol-Dependent Patients. Subst. Use Misuse 2017, 52, 108-118. [CrossRef] 
27. Halari, R.; Premkumar, P.; Farquharson, L.; Fannon, D.; Kuipers, E.; Kumari, V. Rumination and Negative Symptoms in Schizophrenia. J. Nerv. Ment. Dis. 2009, 197, 703-706. [CrossRef]

28. Cooper, Z.; Fairburn, C. The eating disorder examination: A semi-structured interview for the assessment of the specific psychopathology of eating disorders. Int. J. Eat. Disord. 1987, 6, 1-8. [CrossRef]

29. Fairburn, C.G.; Cooper, Z.; Shafran, R. Cognitive behaviour therapy for eating disorders: A "transdiagnostic" theory and treatment. Behav. Res. Ther. 2003, 41, 509-528. [CrossRef]

30. Smith, K.E.; Mason, T.B.; Lavender, J.M. Rumination and eating disorder psychopathology: A meta-analysis. Clin. Psychol. Rev. 2018, 61, 9-23. [CrossRef]

31. Napolitano, M.A.; Himes, S. Race, weight, and correlates of binge eating in female college students. Eat. Behav. 2011, 12, 29-36. [CrossRef] [PubMed]

32. Sassaroli, S.; Bertelli, S.; DeCoppi, M.; Crosina, M.; Milos, G.; Ruggiero, G. Worry and eating disorders: A psychopathological association. Eat. Behav. 2005, 6, 301-307. [CrossRef]

33. Sternheim, L.; Startup, H.; Saeidi, S.; Morgan, J.; Hugo, P.; Russell, A.; Schmidt, U. Understanding catastrophic worry in eating disorders: Process and content characteristics. J. Behav. Ther. Exp. Psychiatry 2012, 43, 1095-1103. [CrossRef]

34. Liberati, A.; Altman, D.G.; Tetzlaff, J.; Mulrow, C.; Gøtzsche, P.C.; Ioannidis, J.P.A.; Clarke, M.; Devereaux, P.J.; Kleijnen, J.; Moher, D. The PRISMA statement for reporting systematic reviews and meta-analyses of studies that evaluate healthcare interventions: Explanation and elaboration. J. Clin. Epidemiol. 2009, 62, e1-e34. [CrossRef]

35. American Psychiatric Association (APA). Diagnostic and Statistical Manual of Mental Disorders, 3rd ed.; American Psychiatric Association: Washington, DC, USA, 1980.

36. American Psychiatric Association (APA). Diagnostic and Statistical Manual of Mental Disorders, 3rd ed.; American Psychiatric Association: Washington, DC, USA, 1987.

37. American Psychiatric Association (APA). Diagnostic and Statistical Manual of Mental Disorders, 4th ed.; American Psychiatric Association: Washington, DC, USA, 1994.

38. American Psychiatric Association (APA). Diagnostic and Statistical Manual of Mental Disorders, 4th ed.; American Psychiatric Association: Washington, DC, USA, 2000.

39. American Psychiatric Association (APA). Diagnostic and Statistical Manual of Mental Disorders, 5th ed.; American Psychiatric Association: Washington, DC, USA, 2013.

40. Spitzer, R.L.; Endicott, J.; Robins, E. Research Diagnostic Criteria: Rationale and reliability. Arch. Gen. Psychiatry 1978, $35,773$. [CrossRef] [PubMed]

41. World Health Organization. Manual of the 28. International Statistical Classification of Diseases, Injuries, and Causes of Death; Sixth Revision of the International Lists of Diseases and Causes of Death, Adopted 1948; World Health Organization: Geneva, Switzerland, 1948.

42. World Health Organization. Manual of the 30. International Statistical Classification of Diseases, Injuries, and Causes of Death; Based on the Recommendations of the Seventh Revision Conference, 1955, and Adopted by the Ninth World Health Assembly under the WHO Nomenclature Regulations; World Health Organization: Geneva, Switzerland, 1957.

43. World Health Organization. Manual of the 32. International Statistical Classification of Diseases, Injuries, and Causes of Death; Based on Recommendations of the Eighth Revision Conference, 1965, and Adopted by the Nineteenth World Health Assembly; World Health Organization: Geneva, Switzerland, 1967.

44. World Health Organization. Manual of the 34. International Classification of Diseases, Injuries, and Causes of Death; Based on the Recommendations of the Ninth Revision Conference, 1975, and Adopted by the Twenty-Ninth World Health Assembly; World Health Organization: Geneva, Switzerland, 1977.

45. World Health Organization. The ICD-10 Classification of Mental and Behavioural Disorders: Clinical Descriptions and Diagnostic Guidelines; World Health Organization: Geneva, Switzerland, 1992.

46. Mansueto, G.; Caselli, G.; Ruggiero, G.M.; Sassaroli, S. Metacognitive beliefs and childhood adversities: An overview of the literature. Psychol. Health Med. 2018, 24, 542-550. [CrossRef]

47. Zhu, C.; Sun, X.; So, S.H.-W. Associations between belief inflexibility and dimensions of delusions: A meta-analytic review of two approaches to assessing belief flexibility. Br. J. Clin. Psychol. 2018, 57, 59-81. [CrossRef]

48. Palmieri, S.; Mansueto, G.; Ruggiero, G.M.; Caselli, G.; Sassaroli, S.; Spada, M.M. Metacognitive beliefs across eating disorders and eating behaviours: A systematic review. Clin. Psychol. Psychother. 2021. [CrossRef]

49. Herzog, R.; Álvarez-Pasquin, M.J.; Díaz, C.; Del Barrio, J.L.; Estrada, J.M.; Gil, Á. Are healthcare workers' intentions to vaccinate related to their knowledge, beliefs and attitudes? A systematic review. BMC Public Health 2013, 13, 154. [CrossRef]

50. Wells, G.; Shea, B.; O'Connell, D.; Peterson, J.E.; Welch, V.; Losos, M.; Tugwell, P. The Newcastle-Ottawa Scale (NOS) for Assessing the Quality of Non-Randomized Studies in Meta-Analysis; Ottawa Hospital Research Institute: Ottawa, ON, Canada, 2010. Available online: http:/ / www.ohri.ca/programs/clinical_epidemiology/oxford.asp (accessed on 16 March 2021). [CrossRef]

51. Hakamata, Y.; Lissek, S.; Bar-Haim, Y.; Britton, J.C.; Fox, N.A.; Leibenluft, E.; Ernst, M.; Pine, D.S. Attention Bias Modification Treatment: A Meta-Analysis Toward the Establishment of Novel Treatment for Anxiety. Biol. Psychiatry 2010, 68, 982-990. [CrossRef] [PubMed]

52. Scaini, S.; Belotti, R.; Ogliari, A.; Battaglia, M. A comprehensive meta-analysis of cognitive-behavioral interventions for social anxiety disorder in children and adolescents. J. Anxiety Disord. 2016, 42, 105-112. [CrossRef] [PubMed] 
53. Duval, S.; Tweedie, R. Trim and Fill: A Simple Funnel-Plot-Based Method of Testing and Adjusting for Publication Bias in Meta-Analysis. Biometrics 2000, 56, 455-463. [CrossRef] [PubMed]

54. Rosenthal, R. The file drawer problem and tolerance for null results. Psychol. Bull. 1979, 86, 638. [CrossRef]

55. Juffer, F.; Van Ijzendoorn, M.H. Adoptees do not lack self-esteem: A meta-analysis of studies on self-esteem of transracial, international, and domestic adoptees. Psychol. Bull. 2007, 133, 1067. [CrossRef] [PubMed]

56. Borenstein, M.; Hedges, L.V.; Higgins, J.; Rothstein, H.R. References; John Wiley \& Sons, Ltd.: Hoboken, NJ, USA, 2009; pp. 409-414.

57. González, M.; Ibáñez, I.; Barrera, A. Rumination, worry and negative problem orientation: Transdiagnostic processes of anxiety, eating behavior and mood disorders. Acta Colomb. Psicol. 2017, 20, 42-52.

58. Kollei, I.; Brunhoeber, S.; Rauh, E.; De Zwaan, M.; Martin, A. Body image, emotions and thought control strategies in body dysmorphic disorder compared to eating disorders and healthy controls. J. Psychosom. Res. 2012, 72, 321-327. [CrossRef]

59. Naumann, E.; Tuschen-Caffier, B.; Voderholzer, U.; Caffier, D.; Svaldi, J. Rumination but not distraction increases eating-related symptoms in anorexia and bulimia nervosa. J. Abnorm. Psychol. 2015, 124, 412. [CrossRef] [PubMed]

60. Sapuppo, W.; Ruggiero, G.M.; Caselli, G.; Sassaroli, S. The Body of Cognitive and Metacognitive Variables in Eating Disorders: Need of Control, Negative Beliefs about Worry Uncontrollability and Danger, Perfectionism, Self-esteem and Worry. Isr. J. Psychiatry Relat. Sci. 2018, 55, 55-63.

61. Troop, N.A.; Treasure, J.L. Psychosocial factors in the onset of eating disorders: Responses to life-events and difficulties. Br. J. Med. Psychol. 1997, 70, 373-385. [CrossRef]

62. Cowdrey, F.A.; Park, R.J. The role of experiential avoidance, rumination and mindfulness in eating disorders. Eat. Behav. 2012, 13, 100-105. [CrossRef]

63. Rawal, A.; Park, R.J.; Williams, J.M.G. Rumination, experiential avoidance, and dysfunctional thinking in eating disorders. Behav. Res. Ther. 2010, 48, 851-859. [CrossRef] [PubMed]

64. Wang, S.B.; Borders, A. The unique effects of angry and depressive rumination on eating-disorder psychopathology and the mediating role of impulsivity. Eat. Behav. 2018, 29, 41-47. [CrossRef] [PubMed]

65. Mason, T.B.; Lewis, R.J. Examining social support, rumination, and optimism in relation to binge eating among Caucasian and African-American college women. Eat. Weight. Disord. 2017, 22, 693-698. [CrossRef]

66. Startup, H.; Lavender, A.; Oldershaw, A.; Stott, R.; Tchanturia, K.; Treasure, J.; Schmidt, U. Worry and Rumination in Anorexia Nervosa. Behav. Cogn. Psychother. 2013, 41, 301-316. [CrossRef]

67. Zarychta, K.; Mullan, B.; Kruk, M.; Luszczynska, A. A vicious cycle among cognitions and behaviors enhancing risk for eating disorders. BMC Psychiatry 2017, 17, 154. [CrossRef]

68. Crino, N.; Touyz, S.; Rieger, E. How eating disordered and non-eating disordered women differ in their use (and effectiveness) of cognitive self-regulation strategies for managing negative experiences. Eat. Weight. Disord. 2019, 24, 897-904. [CrossRef] [PubMed]

69. Hartmann, A.S.; Cordes, M.; Hirschfeld, G.; Vocks, S. Affect and worry during a checking episode: A comparison of individuals with symptoms of obsessive-compulsive disorder, anorexia nervosa, bulimia nervosa, body dysmorphic disorder, illness anxiety disorder, and panic disorder. Psychiatry Res. 2019, 272, 349-358. [CrossRef]

70. Connolly, A.M.; Rieger, E.; Caterson, I. Binge eating tendencies and anger coping: Investigating the confound of trait neuroticism in a non-clinical sample. Eur. Eat. Disord. Rev. 2007, 15, 479-486. [CrossRef]

71. Nolen-Hoeksema, S.; Stice, E.; Wade, E.; Bohon, C. Reciprocal relations between rumination and bulimic, substance abuse, and depressive symptoms in female adolescents. J. Abnorm. Psychol. 2007, 116, 198-207. [CrossRef] [PubMed]

72. Harrell, Z.A.T.; Jackson, B. Thinking Fat and Feeling Blue: Eating Behaviors, Ruminative Coping, and Depressive Symptoms in College Women. Sex Roles 2008, 58, 658-665. [CrossRef]

73. Selby, E.A.; Anestis, M.D.; Joiner, T.E. Understanding the relationship between emotional and behavioral dysregulation: Emotional cascades. Behav. Res. Ther. 2008, 46, 593-611. [CrossRef]

74. Aldao, A.; Nolen-Hoeksema, S. Specificity of cognitive emotion regulation strategies: A transdiagnostic examination. Behav. Res. Ther. 2010, 48, 974-983. [CrossRef]

75. Holm-Denoma, J.M.; Hankin, B.L. Perceived Physical Appearance Mediates the Rumination and Bulimic Symptom Link in Adolescent Girls. J. Clin. Child Adolesc. Psychol. 2010, 39, 537-544. [CrossRef] [PubMed]

76. Verplanken, B.; Tangelder, Y. No body is perfect: The significance of habitual negative thinking about appearance for body dissatisfaction, eating disorder propensity, self-esteem and snacking. Psychol. Health 2011, 26, 685-701. [CrossRef] [PubMed]

77. Gordon, K.H.; Holm-Denoma, J.M.; Troop-Gordon, W.; Sand, E. Rumination and body dissatisfaction interact to predict concurrent binge eating. Body Image 2012, 9, 352-357. [CrossRef] [PubMed]

78. Kelly, N.R.; Lydecker, J.; Mazzeo, S.E. Positive cognitive coping strategies and binge eating in college women. Eat. Behav. 2012, 13, 289-292. [CrossRef]

79. Hilt, L.M.; Roberto, C.A.; Nolen-Hoeksema, S. Rumination mediates the relationship between peer alienation and eating pathology in young adolescent girls. Eat. Weight. Disord. 2013, 18, 263-267. [CrossRef]

80. Svaldi, J.; Naumann, E. Effects of Rumination and Acceptance on Body Dissatisfaction in Binge Eating Disorder. Eur. Eat. Disord. Rev. 2014, 22, 338-345. [CrossRef] 
81. Mason, T.B.; Lewis, R.J. Minority Stress and Binge Eating Among Lesbian and Bisexual Women. J. Homosex. 2015, 62, 971-992. [CrossRef]

82. Breithaupt, L.; Rallis, B.; Mehlenbeck, R.; Kleiman, E. Rumination and self-control interact to predict bulimic symptomatology in college students. Eat. Behav. 2016, 22, 1-4. [CrossRef]

83. Jungmann, S.M.; Vollmer, N.; Selby, E.A.; Witthöft, M. Understanding Dysregulated Behaviors and Compulsions: An Extension of the Emotional Cascade Model and the Mediating Role of Intrusive Thoughts. Front. Psychol. 2016, 7, 994. [CrossRef]

84. Maraldo, T.M.; Zhou, W.; Dowling, J.; Wal, J.S.V. Replication and extension of the dual pathway model of disordered eating: The role of fear of negative evaluation, suggestibility, rumination, and self-compassion. Eat. Behav. 2016, 23, 187-194. [CrossRef]

85. Naumann, E.; Tuschen-Caffier, B.; Voderholzer, U.; Svaldi, J. Spontaneous Emotion Regulation in Anorexia and Bulimia Nervosa. Cogn. Ther. Res. 2016, 40, 304-313. [CrossRef]

86. Seidel, M.; Petermann, J.; Diestel, S.; Ritschel, F.; Boehm, I.; King, J.A.; Geisler, D.; Bernardoni, F.; Roessner, V.; Goschke, T.; et al. A naturalistic examination of negative affect and disorder-related rumination in anorexia nervosa. Eur. Child Adolesc. Psychiatry 2016, 25, 1207-1216. [CrossRef]

87. Opwis, M.; Salewski, C.; Schmidt, J.; Martin, A. Gender differences in eating behavior and eating pathology: The mediating role of rumination. Appetite 2017, 110, 103-107. [CrossRef]

88. Wang, S.B.; Borders, A. Rumination mediates the associations between sexual minority stressors and disordered eating, particularly for men. Eat. Weight. Disord. 2017, 22, 699-706. [CrossRef]

89. Wang, S.B.; Lydecker, J.A.; Grilo, C.M. Rumination in Patients with Binge-Eating Disorder and Obesity: Associations with Eating-Disorder Psychopathology and Weight-bias Internalization. Eur. Eat. Disord. Rev. 2017, 25, 98-103. [CrossRef]

90. Dondzilo, L.; Rieger, E.; Palermo, R.; Byrne, S.; Bell, J. Correction: The mediating role of rumination in the relation between attentional bias towards thin female bodies and eating disorder symptomatology. PLoS ONE 2018, 13, e0196143. [CrossRef]

91. Van Durme, K.; Goossens, L.; Bosmans, G.; Braet, C. The Role of Attachment and Maladaptive Emotion Regulation Strategies in the Development of Bulimic Symptoms in Adolescents. J. Abnorm. Child Psychol. 2017, 46, 881-893. [CrossRef]

92. Birmachu, A.M.; Heidelberger, L.; Klem, J. Rumination and perceived social support from significant others interact to predict eating disorder attitudes and behaviors in university students. J. Am. Coll. Health 2019. [CrossRef]

93. Fresnics, A.A.; Wang, S.B.; Borders, A. The unique associations between self-compassion and eating disorder psychopathology and the mediating role of rumination. Psychiatry Res. 2019, 274, 91-97. [CrossRef]

94. Hernando, A.; Pallás, R.; Cebolla, A.; García-Campayo, J.; Hoogendoorn, C.J.; Roy, J.F. Mindfulness, rumination, and coping skills in young women with Eating Disorders: A comparative study with healthy controls. PLoS ONE 2019, 14, e0213985. [CrossRef]

95. Smith, K.E.; Mason, T.B.; Anderson, N.L.; Lavender, J.M. Unpacking cognitive emotion regulation in eating disorder psychopathology: The differential relationships between rumination, thought suppression, and eating disorder symptoms among men and women. Eat. Behav. 2019, 32, 95-100. [CrossRef] [PubMed]

96. Branley-Bell, D.; Talbot, C.V. Exploring the impact of the COVID-19 pandemic and UK lockdown on individuals with expe-rience of eating disorders. J. Eat. Disord. 2020, 8, 1-12. [CrossRef]

97. Martino, F.; Caselli, G.; Berardi, D.; Fiore, F.; Marino, E.; Menchetti, M.; Prunetti, E.; Ruggiero, G.M.; Sasdelli, A.; Selby, E.; et al. Anger rumination and aggressive behaviour in borderline personality disorder. Pers. Ment. Health 2015, 9, 277-287. [CrossRef]

98. Salters-Pedneault, K.; Roemer, L.; Tull, M.; Rucker, L.; Mennin, D.S. Evidence of Broad Deficits in Emotion Regulation Associated with Chronic Worry and Generalized Anxiety Disorder. Cogn. Ther. Res. 2006, 30, 469-480. [CrossRef]

99. Vann, A.; Strodl, E.; Anderson, E. Thinking about internal states, a qualitative investigation into metacognitions in women with eating disorders. J. Eat. Disord. 2013, 1, 22. [CrossRef]

100. Vinai, P.; Da Ros, A.; Cardetti, S.; Casey, H.; Studt, S.; Gentile, N.; Tagliabue, A.; Vinai, L.; Vinai, P.; Bruno, C.; et al. The DSM-5 effect: Psychological characteristics of new patients affected by Binge Eating Disorder following the criteria of the DSM-5 in a sample of severe obese patients. Eat. Weight Disord. 2016, 21, 107-113. [CrossRef] [PubMed]

101. Wells, A.; Matthews, G. Modelling cognition in emotional disorder: The S-REF model. Behav. Res. Ther. 1996, 34, 881-888. [CrossRef]

102. Wells, A. Emotional Disorders and Metacognition: Innovative Cognitive Therapy; Wiley: Chichester, UK, 2000.

103. Spada, M.M.; Caselli, G.; Nikčević, A.V.; Wells, A. Metacognition in addictive behaviors. Addict. Behav. 2015, 44, 9-15. [CrossRef]

104. Spada, M.M.; Caselli, G.; Fernie, B.A.; Manfredi, C.; Boccaletti, F.; Dallari, G.; Gandini, F.; Pinna, E.; Ruggiero, G.M.; Sassaroli, S. Desire thinking: A risk factor for binge eating? Eat. Behav. 2015, 18, 48-53. [CrossRef]

105. Mansueto, G.; Martino, F.; Palmieri, S.; Scaini, S.; Ruggiero, G.M.; Sassaroli, S.; Caselli, G. Desire Thinking across addictive behaviours: A systematic review and meta-analysis. Addict. Behav. 2019, 98, 106018. [CrossRef]

106. Hong, R.Y. Worry and rumination: Differential associations with anxious and depressive symptoms and coping behavior. Behav. Res. Ther. 2007, 45, 277-290. [CrossRef]

107. Hoyer, J.; Gloster, A.T.; Herzberg, P.Y. Is worry different from rumination? Yes, it is more predictive of psychopathology! GMS Psycho Soc. Med. 2009, 6. [CrossRef]

108. Kaplan, D.M.; Palitsky, R.; Carey, A.L.; Crane, T.E.; Havens, C.M.; Medrano, M.R.; Reznik, S.J.; Sbarra, D.A.; O'Connor, M.-F. Maladaptive repetitive thought as a transdiagnostic phenomenon and treatment target: An integrative review. J. Clin. Psychol. 2018, 74, 1126-1136. [CrossRef] [PubMed]

109. Watkins, E.R. Rumination-Focused Cognitive-Behavioral Therapy for Depression; Guilford Publications: New York, NY, USA, 2018. 
110. Fava, G.A. Meta-Analyses and Conflict of Interest. CNS Drugs 2012, 26, 93-96. [CrossRef] [PubMed]

111. Cowdrey, F.A.; Park, R.J. Assessing rumination in eating disorders: Principal component analysis of a minimally modified ruminative response scale. Eat. Behav. 2011, 12, 321-324. [CrossRef] 